\title{
ENERGY MACHINERIES ON A MANIFOLD; APPLICATION TO THE CONSTRUCTION OF NEW ENERGY REPRESENTATIONS OF GAUGE GROUPS*
}

\author{
J. MAIUION
}

\begin{abstract}
The introduction of the concepts of encrgy maclinery and energy struclure on a manifold makes it possible a large class of energy representations of gauge groups including, as a very particular case, the ones known up to now. By using an adaptation of methods iniliated by I.M. Gelfand, we provide a sufficient condition for the irreducibility of these representations.
\end{abstract}

\section{Introduction}

a) Let $X$ be a smooth Riemannian manifold, let $G$ be a compact semisimple Lie group with Lie algebra $g$, let $\mathcal{D}(X, G)$ be the gauge group consisting of all the $G$-valued and compactly supported smooth mappings on $X$, let $\mathcal{D}_{1}(X, g)$ be the nuclear space of all the $\mathcal{G}$-valued and compactly supported smooth 1 . forms on $X$, and let $b: \mathcal{D}(X, G) \rightarrow \mathcal{D}_{1}(X, g)$ be the Maurer-Cartan cocycle $g \rightarrow b(g)=d g \cdot g^{-1}$.

An energy representation of $\mathcal{D}(X, G)$ is a unitary representation $\Pi$ of $\mathcal{D}(X, G)$ into the symmetric Fock space of some complex Hilbert space $(h, \|||)$ such that:

(1) there exists a continuous morphism $\Phi$ from $\mathcal{D}_{1}(X, \mathcal{G})$ into $h$;

(2) the spherical function $\varphi$ of $\Pi$ with respect to the vacuum vector EXPO is given by

$$
g \longrightarrow \varphi(g)=<\Pi(g) \operatorname{EXPO}, \operatorname{EXPO}>=\exp \left\{-\frac{1}{2} \| \Phi\left(b(g) \|^{2}\right\} .\right.
$$

* Supported in part by the Centre National de la Recherche Scientifielue (C.N.R.S.) and by the Deutsche Forschungsgemeinschaft (DFG). 
b) In [11] I have exposed why, from several points of view, such representations of gauge groups are interesting; at that time only two kinds of energy representations were known: those constructed by Ismagilov [7] and Gelfand et al. [16], [17] associated with Riemannian structures on $X$, and the ones constructed in [9] which are associated to non vanishing vector fields on $X$ (for more details, sce the Historical Note in $\$ 7$ ).

c) The present paper tries to bring a substantial answer (although incomplete) to the open questions exposed in [11]. More precisely, we introduce the concepts of energy machinery and of measurable field of such objects, which allow to endow some spaces related to $\mathcal{D}_{1}(X, g)$ with $\mathcal{D}(X, G)$-invariant Euclidean structures, namely the energy structures. Then, the Maurer-Cartan cocycle $b$ and the method exposed in $[6],[7],[17]$ allow to associate to each energy structurc an energy representation of $\mathcal{D}(X, G)$ (Theorem 1 ).

d) The last part of the paper $(\S \S 7,8,9)$ is devoted to extend the papers $[\mathbf{1 6}]$, $[17],[18]$ and $[2]$ in order to get a sufficient condition for the irreducibility of the energy representations with support $X$, the so-called $\Gamma$-property, which turns the irreducibility into a result about a kind of gtrong disjointness of Gaussian measures closely connected with the restriction of the energy representations to $\mathcal{D}(X, T)$, where $T$ is some Cartan subgroup of $G$ (Theorem 3 ).

\section{Preliminaries and Notations}

a) In all this paper $X$ is a $n$-dimensional smooth Riemannian manifold; as usual, $T X$ specifies its tangent bundle, and $T^{*} X$ its contangent bundle; $\mathcal{D}\left(T^{*} X\right)$ is the space of real valued and compactly supported smooth 1 -forms on $X$ endowed with the Schwartz topology of compactly supported smooth sections; it is well-known that $\mathcal{D}\left(T^{*} X\right)$ is a $L F$-space, that is to say a real nuclear space, inductive limit of Fréchet spaces (see e.g. [15]).

b) The set $\mathcal{R}(X)$ of Riemannian structures on $X$ is by definition the subset of $C^{\infty}\left(S^{2} T^{*} X\right)$ consisting of sections $\tau$ which induce a positive definite inner product $q_{r_{1} x}$ on each tangent space $T_{x} X$, and therefore a volume measure $d v^{r}$ on $X$. Let $\tau$ be in $R(X)$ and let $r$ be in the set $C_{+}^{1}(X)$ of all the strictly positive $C^{1}$-functions on $X$; an elemert $x$ of $X$ being given, one gets a positive definite inner product $q_{\tau, x}^{*}$ on the cotangent space $T_{x}^{*} X$ by

$$
q_{\tau, x}^{*}\left(u_{x}, v_{x}\right)=\operatorname{tr}\left(u_{x}^{*}, v_{x}\right),\left(u_{x}, v_{x}\right) \in\left(T_{x}^{*} X\right)^{2},
$$

where $u_{x}^{*}: \mathbb{R} \rightarrow T_{x} X$ denotes the adjoint of $u_{x}$ with respect to $q_{\tau_{x}}$. One gets also a positive definite inner product ( , ) $\tau, r$ on the space $\mathcal{D}\left(T^{*} X\right)$ of all the compactly supported real smooth 1 -forms on $X$, given by:

$$
\left(\omega, \omega^{\prime}\right)_{\tau, r}=\int_{X} q_{\tau, 2}^{*}\left(\omega(x), \omega^{\prime}(x)\right) r(x) d v^{\tau}(x) .
$$


c) Let $F=\underset{x \in X}{\bigcup} F_{x}$ be a smooth subbundle of $T X$, and let $Y$ be a connected smooth submanifold of $X . F^{Y}$ will be the restricted subbundle $\underset{x \in Y}{\bigcup} F_{x}$, and $M^{+}(Y)$ will be the set of strictly positive measures on $Y$. An element $\omega$ in $\mathcal{D}\left(T^{*} X\right)$ being given, $\omega^{F}$ will be the mapping from $X$ into the dual bundle $F^{*}=\underset{x \in X}{\bigcup} F_{x}^{*}$ of $F$, such that for all $x$ in $X, \omega^{F}(x)$ is the restriction of $\omega(x)$ to $F_{x}$, and $\omega_{Y}^{F}$ will be the restriction of $\omega^{F}$ to the submanifold $Y$. An euclidean structure $q$ on $F^{Y}$ is a family $q=\left(q_{x}\right)_{x \in Y}$ such that for all $x$ in $Y, q_{x}$ is a scalar product on $F_{x}$; of course, one gets a scalar product $q_{x}^{*}$ on $F_{z}^{*}, x \in Y$, by:

$$
q_{x}^{*}\left(u_{x}, v_{x}\right)=\operatorname{tr}\left(u_{x}^{*} \cdot v_{x}\right),\left(u_{x}, v_{x}\right) \in F_{x}^{*} x F_{x}^{*},
$$

where $u_{x}^{*}$ denotes the adjoint of $u_{x}$ with respect to the scalar product $q_{x}$ on $F_{x}$.

d) Let $\tau$ be an element of $\mathcal{R}(X)$, let $F$ be a smooth subbundle of $T X$, and let $Y$ be a submanifold of $X . F^{\perp}=\bigcup_{x \in X} F_{x}^{\perp}$, where $F_{x}^{\perp}$ is the orthogonal complement of $F_{x}$ with respect to the scalar product $q_{\tau, r}$ will be called the $\tau$-orthogonal complement of $F$, and we have a canonical identification of the space $\left\{\omega^{F} / \omega \in \mathcal{D}\left(T^{*} X\right)\right.$ with the subspace of $\mathcal{D}\left(T^{*} X\right)$ :

$$
\mathcal{D}\left(T^{*} X\right)^{F}=\left\{\omega \in \mathcal{D}\left(T^{*} X\right) / \omega^{F^{\perp}}=0\right\} .
$$

From proposition 50.1 of $[\mathbf{1 5}]$ it follows that $\mathcal{D}\left(T^{*} X\right)^{F}$, as subspace of $\mathcal{D}\left(T^{*} X\right)$, is a real nuclear space, inductive limit of Fréchet spaces.

$\mathcal{D}\left(T^{*} X\right)_{Y}^{F}$ will be the space $\left\{\omega_{Y}^{F} / \omega^{F} \in \mathcal{D}\left(T^{*} X\right)^{F}\right\}$; one has also:

$$
\mathcal{D}\left(T^{*} Z\right)_{Y}^{F}=\left\{\omega_{Y}^{F} / \omega \in \mathcal{D}\left(T^{*} X\right)\right\} .
$$

\section{Energy Machineries on the Manifold $X$}

Definition 1. An energy machinery on the manifold $X$ is a quadruplet $\varepsilon=(Y, d \nu, F, q)$ such that:

(1) $Y$ is a connected submanifold of $X$ endowed with a strictly positive measure $d \nu$

(2) $F$ is a smooth subbundle of $T X$, and $q=\left(q_{x}\right)_{x \in Y}$ is an Euclidean structure on $F^{Y}$;

(3) for all $\omega, \omega^{\prime}$ in $\mathcal{D}\left(T^{*} X\right)$ the mapping:

$$
x \longrightarrow q_{x}^{*}\left(\omega^{F}(x), \omega^{i F}(x)\right), \quad x \in Y
$$

is $d \nu$-integrable.

An obvious example of energy machinery is the following:

Let $\tau$ be in $R(X)$, and let $\rho$ be in $C_{+}^{1}(X)$; it follows from (1) that $\left(X, \rho d v^{\tau}\right.$, $\left.T X,\left(q_{r, x}\right)_{x \in X}\right)$ is an energy machinery. We shall give later on numerous other examples. 
Lemma 1. Let $\varepsilon=(Y, d \nu, F, q)$ be an energy machinery on $X$.

(i) The mapping: $\left(\omega_{Y}^{F}, \omega_{Y}^{\prime F}\right) \rightarrow\left(\omega_{Y}^{F}, \omega_{Y}^{\prime F}\right)_{\varepsilon}=\int_{Y} q_{x}^{*}\left(\omega^{F}(x), \omega^{\prime F}(x)\right) d \nu(x)$ is a positive definite inner product on $\mathcal{D}\left(T^{*} X\right)_{Y}^{F}$;

(ji) the mapping: $\left(\omega, \omega^{\prime}\right) \rightarrow\left(\omega, \omega^{\prime}\right)_{\varepsilon}=\left(\omega_{Y}^{F}, \omega_{Y}^{\prime F}\right)_{\varepsilon}$ is a positive inner product on $\mathcal{D}\left(T^{*} X\right)$.

(iii) the mapping: $\left(\omega^{F}, \omega^{\prime F}\right) \rightarrow\left(\omega_{Y}^{F}, \omega_{Y}^{\prime F}\right)_{\varepsilon}$ is a positive inner product on $\mathcal{D}\left(T^{*} X\right)^{F}$.

Proof: The lemma is an obvious consequence of the above definition.

\section{Complete ${ }^{-}$-Energy Machineries on $X$}

a) Definition 2. Let $\tau$ be in $\mathcal{R}(X)$; the energy machineries of the form $\varepsilon=\left(X, r d v^{\top}, F, q\right)$ are called the complete $\tau$-energy machineries on $X$.

From Lemma 1, it follows easily:

Lemma 2. Let $\varepsilon$ be a complete $r$-energy machinery of the form $\varepsilon=\left(X, r d v^{\top}\right.$, $T X, q)$. The mapping:

$$
\left(\omega, \omega^{\prime}\right) \rightarrow\left(\omega, \omega^{\prime}\right)_{\epsilon}=\int_{X} q_{x}^{*}\left(\omega(x), \omega^{\prime}(x)\right) r(x) d v^{\top}(x)
$$

is a positive definite inner product on $D\left(T^{*} X\right)$.

Definition 3. The complete $\tau$-energy machineries of the form $\varepsilon=\left(X, r d v^{\tau}\right.$, $T X, q)$ are called regular complete $\tau$-energy machineries.

$\left(X, r d v^{\tau}, T X,\left(q_{\tau, x}\right)_{x \in X}\right)$ is the basic example of regular complete $\tau$-energy machineries and for a given subbundle $F \neq(0)$ of $T X,\left(X, r d v^{r}, F,\left(q_{\tau, x}\right)_{x \in X}\right)$ is a basic example of complete $\tau$-energy machineries.

We have to give now other examples.

b) Let us suppose that the Euler number $e(X)$ of $X$ is zero; as it is wellknown (see e.g. [14], $§ 39.8$ ) we can find an integer $k, 1 \leq k \leq n$, and a $k$-frame:

$$
\xi=\left(\xi_{1}, \ldots, \xi_{k}\right)
$$

of smooth vector fields on $X$ which generates a smooth subbundle:

$$
F(\xi)=\bigcup_{x \in X} F_{x}(\xi) \text { with } F_{x}(\xi)=\underset{i=1}{\oplus=k} \mathbb{R} \xi_{i}(x) ;
$$

we endow $F(\xi)$ with the Euclidean structure $q^{\xi}=\left(q_{x}^{\xi}\right)_{x \in X}$ such that:

$$
q_{x}\left(\sum_{i=1}^{i=k} a_{i} \xi_{i}(x), \sum_{i=1}^{i=k} b_{i} \xi_{i}(x)\right)=\sum_{i=1}^{i=k} a_{i} b_{i}
$$

it follows that, an element $\tau$ of $R(X)$ and an element $r$ of $C_{+}^{1}(X)$ being given, for all $\omega, \omega^{\prime}$ in $\mathcal{D}\left(T^{*} X\right)$ the mapping:

$$
x \longrightarrow\left(q_{x}^{\xi}\right)^{*}\left(\omega^{F(\xi)}(x), \omega^{t F(\xi)}(x)\right)
$$

is $r d v^{r}$-integrable on $X$. So: 
Lemma 3. Let $\tau$ be in $\mathcal{R}(X)$, let $r$ be in $C_{+}^{1}(X)$, and let us suppose that $X$ has a $k$-frame of smooth vector fields $\xi=\left(\xi_{1}, \ldots, \xi_{k}\right), k \geq 1 .\left(X, r d v^{\tau}, F(\xi), q^{\xi}\right)$ is a complete $\tau$-energy machinery.

c) There exists a universal way to get complete $\tau$-energy machineries: let $\tau$ be in $\mathcal{R}(X)$, let $r$ be in $C_{+}^{1}(X)$ and let $F \neq(0)$ be a smooth subbundle of $T X ; k$ will be the common dimension of the fibers $F_{x}$ of $F$. Let us consider the corresponding infinite Grassmann manifold $G_{k}\left(\mathbb{R}^{\infty}\right)$, and let $\gamma_{k}$ be universal bundle with base $G_{k}\left(\mathbb{R}^{\infty}\right)$ (see e.g. $\left.[12], \S 5.8\right)$. The set of continuous bundle morphisms from $F$ into. $\gamma_{k}$ is not empty ([12], theorem 5.6). Let $\theta$ be such a morphism, and for each $x$ in $X$, let $\theta_{x}$ be the corresponding linear isomorphism from $F_{x}$ onto some $k$-dimensional subspace of $\mathbb{R}^{\infty}, \mathbb{R}^{\infty}$ being endowed with its canonical scalar product $<, \quad>$; one gets a scalar product $g_{x}^{\theta}$ on $F_{x}$ by $q_{x}^{\theta}(u, v)=\left\langle\theta_{x}(u), \theta_{x}(v)>\right.$. Let $q^{\theta}$ be the family $\left(q_{x}^{\theta}\right)_{x \in X}$. From the continuity of $\theta$, it follows that for all $\omega, \omega^{\prime}$ in $\mathcal{D}\left(T^{*} X\right)$ the mapping $x \rightarrow\left(q_{x}^{\theta}\right)^{*}\left(\omega^{F}(x), \omega^{\prime F}(x)\right)$ is $r d v^{T}$-integrable on $X$, and then $\left(X, r d v^{r}, F, q\right)$ is a complete $\tau$-energy machinery.

d) Let $\varepsilon=\left(X, r d v^{\tau}, F, q\right)$ be a complete $r$-energy machinery, and let $\Gamma_{+}^{1}(X)$ be the set of global sections $\sigma$ of class $C^{1}$ of the bundle $\operatorname{Hom}(F, F)$, such that, for all $x$ in $X, \sigma(x)$ is a positive definite operator on $F_{x}$. Each element $\sigma$ of $\Gamma_{+}^{\mathbf{l}}(X)$ gives rise to a new scalar product $\sigma(q)_{x}$ such that $\sigma(q)_{x}(u, v)=$ $q_{x}(\sigma(x) u, \sigma(x) v)$. From the continuity of $\sigma$ it follows that $\left(X, r d v^{r}, F, \sigma(q)\right)$, with $\sigma(q)=\left(\sigma(q)_{x}\right)_{x \in X}$, is a complete $\tau$-energy machinery.

e) Let us suppose that $\operatorname{dim}(X) \geq 2$, let $\tau$ be in $R(X)$, and let $F$ be a proper smooth subbundle of $T X$, i.e. $F \neq(0)$ and $F \neq T X . \varepsilon=\left(X, r d v^{r}, F, q_{\tau}^{F}\right)$ is a complete $\tau$-energy machinery, where $q_{\tau}^{F}$ is the Euclidean structure coming from $\tau$ and restricted to $F$. Now let us consider the orthogonal subbundle $F^{\perp}$, and let $\tau^{\prime}$ be another Riemannian structure on $X ; q_{\tau^{\prime}}^{F^{\perp}}$ will denote the restriction to $F^{\perp}$ of the Euclidean structure coming from $\tau^{\prime}$.

One gets a new Euclidean structure $q^{r, F, r^{\prime}}=\left(q_{x}^{\tau, F, r^{\prime}}\right)_{x \in X}$ on $T X=F \oplus F^{\perp}$ such that, for all $x$ in $X$ :

$$
q_{x}^{\tau, F, \tau^{\prime}}\left(u_{x}+u_{x}^{\perp}, v_{x}+v_{x}^{\perp}\right)=q_{\tau, x}^{F}\left(u_{x}, v_{x}\right)+q_{r^{\prime}, x}^{F^{\perp}}\left(u_{y}^{\perp}, v_{x}^{\perp}\right)
$$

with $\left(u_{x}, v_{x}\right)$ in $F_{x} x F_{x}$, and $\left(u_{x}^{\perp}, v_{x}^{\perp}\right)$ in $F_{x}^{\perp} x F_{x}^{\perp}$.

From the fact that one has the orthogonal sum:

$$
\mathcal{D}\left(T^{*} X\right)=\mathcal{D}\left(T^{*} X\right)^{F} \oplus F\left(T^{*} X\right)^{F^{\lrcorner}}
$$

with respect to $g_{r}$, corresponding to the decomposition $\omega \rightarrow \omega^{F}+\omega^{F^{\perp}}$, it follows that for all pair $\left(\omega, \omega^{\prime}\right)$ of elements in $D\left(T^{*} X\right)$, and each $x$ in $X$ one has:

$$
\left(q_{x}^{\tau, F, r^{\prime}}\right)^{*}\left(\omega(x), \omega^{\prime}(x)\right)=\left(q_{r, x}^{F}\right)^{*}\left(\omega^{F}(x), \omega^{\prime F}(x)\right)+\left(q_{r^{\prime}, x}^{F^{\perp}}\right)^{*}\left(\omega^{F^{\perp}}(x), \omega^{\prime F^{\perp}}(x)\right)
$$


As $\left(X, r d v^{\tau}, F, q_{\tau}^{F}\right)$ is an energy machinery, the mapping $x \rightarrow\left(q_{\tau, x}^{F}\right)^{*}\left(\omega^{F}(x)\right.$, $\left.\omega^{\prime F}(x)\right)$ is $r d v^{r}$-integrable on $X$; for the same reason, the mapping

$$
x \longrightarrow\left(q_{r^{\prime}, x}^{F^{\perp}}\right)^{*}\left(\omega^{F^{\perp}}(x), \omega^{\prime F^{\perp}}(x)\right) \text { is } d v^{r^{\prime}} \text {-integrable on } X
$$

As $d v^{\tau}$ and $d v^{\tau^{\prime}}$ are smooth strictly positive measures, there exists a strictly positive $C^{\infty}$-function $\lambda$ on $X$ such that $d v^{r}=\lambda d v^{r^{\prime}}$; it follows that:

$$
x \longrightarrow\left(q_{\tau^{1}, x}^{F^{\perp}}\right)^{*}\left(\omega^{F^{\perp}}(x), \omega^{\prime F^{\perp}}(x)\right) \text { is } r d v^{\tau} \text {-integrable on } X
$$

and then

$$
x \longrightarrow\left(q_{x}^{\tau, F, r^{\prime}}\right)^{*}\left(\omega(x), \omega^{\prime}(x)\right) \text { is } r d v^{\tau} \text {-integrable }
$$

From the above study it follows:

Lemma 4. Let $F$ be a proper smooth subbundle of $T X$, with $\operatorname{dim}(X) \geq 2$, and let $\varepsilon=\left(X, r d v^{T}, F, q_{T}^{F}\right)$ be a basic complete $\tau$-energy machinery according to $F$. Another Riemannian structure $\tau^{\prime}$ on $X$ being given, the quadruplet $\varepsilon^{T^{\prime}}=$ $\left(X, r d v^{\tau}, T X, q^{\tau, F, \tau^{\prime}}\right)$ is a regular complete $\tau$-energy machinery. $\varepsilon^{\tau^{\prime}}$ will be called the $\tau^{\prime}$-regularization of $\varepsilon$.

Remark: If $\tau^{\prime}=\tau, q^{\gamma, F, r^{\prime}}=q_{r}$ and then $\varepsilon^{\tau}$ is the basic regular complete $\tau$-energy machinery $\left(X, r d v^{\tau}, T X, q_{\tau}\right)$.

\section{4. $(\Lambda, d \ell$-Measurable Field of Energy Machineries of Type $F$}

a) By standard Borel measure space $(\Lambda, d \ell)$, we mean here a standard Borel space $\Lambda$ endowed with a positive Borel measure $d \ell$ (of course $\sigma$-finite).

Definition 4. Let $(\Lambda, d \ell)$ be a standard Borel measure space, let $F$ be a smooth subbundle of $T X$, and for each $\alpha$ in $\Lambda$, let $\varepsilon_{\alpha}=\left(Y_{\alpha}, d \nu_{\alpha}, F, q_{\alpha}\right)$ be an energy machinery on $X$. The assignment $\alpha \rightarrow \epsilon_{\alpha}$ will be a $(\Lambda, d \ell)$-measurable field of energy machineries of type $F$ if:

(1) $\alpha \neq \alpha^{\prime}$ implies $Y_{\alpha} \cap Y_{\alpha^{\prime}}=\phi$;

(2) $\alpha \rightarrow d \nu_{\alpha}$ is a $d \ell$-integrable field of measures;

(3) for all pair $\left(\omega, \omega^{\prime}\right)$ of elements in $\mathcal{D}\left(T^{*} X\right)$ the thapping $\alpha \rightarrow\left(\omega_{Y_{\alpha}}^{F}, \omega_{Y_{\alpha}}^{F}\right)_{\varepsilon_{\alpha}}$ is $d \ell$-integrable on $\Lambda$;

(4) let $X(\Lambda)=\bigcup_{\alpha \in \Lambda} Y_{\alpha}$; the mapping $\left(\omega_{X(\Lambda)}^{F}, \omega_{X(\Lambda)}^{\prime F}\right) \rightarrow \int_{\Lambda}\left(\omega_{Y_{\alpha}}^{F}, Y_{\alpha}\right) \varepsilon_{\alpha} d \ell(\alpha)$ is a positive definite inner product on $\mathcal{D}\left(T^{*} X\right)_{X(\Lambda)}^{F}$.

Remarks: Let us suppose that $\Lambda$ is reduced to a single point: $\Lambda=\left\{\alpha_{0}\right\}$, endowed with its canonical counting measure $d n_{1}$, and let $\varepsilon=(Y, d \nu, F, q)$ be an energy machinery; the assignment $\alpha \in \Lambda=\left\{\alpha_{0}\right\} \rightarrow \varepsilon_{\alpha}=\varepsilon$ is obviously a $\left(\Lambda, d n_{1}\right)$-measurable field of energy machineries of type $F$; it follows 
that any energy machinery can be viewed as $(\Lambda, d \ell)$-measurable field of energy machineries.

b) Let $\gamma$ be a configuration in $X$, i.e. a non empty locally finite subset of $X$; we can find a subset $\mathbf{N}(\gamma)$ of the set of strictly positive integers $\mathbf{N}^{*}$, with $\mathbb{N}(\gamma)=\{1, \ldots, p\}$ if $\gamma$ is a finite configuration (this is always the case if $X$ is a compact manifold), and with $\mathbf{N}(\gamma)=\mathbf{N}^{*}$ if $\gamma$ is countable, such $i \rightarrow x_{i}$ is a one-to-one mapping from $\mathbf{N}(\gamma)$ onto $\gamma \cdot \mathbf{N}(\gamma)$ is endowed with its counting measure $d n_{\gamma}$, and each subset $\left\{x_{i}\right\}, i$ in $\mathbf{N}(\gamma)$, with its counting measure $d i_{1}$.

Lemma 5. Let $\gamma$ be a configuration in $X$, let $\rho$ be a strictly positive function on $\gamma$, and let $q=\left(q_{i}\right)_{i \in N(\gamma)}$ be a family of scalar products, such that for all $i$ in $\mathbb{N}(\gamma), q_{i}$ is a scalar product on $T_{x_{i}} X$.

The assignment $i \rightarrow \varepsilon_{i}=\left(\left\{x_{i}\right\}, \rho d i_{1}, T X, q_{i}\right)$ is a $\left(\mathbb{N}(\gamma), d n_{\gamma}\right)$-measurable feld of energy machineries of type $T X$.

Proof: Let $i$ be in $\mathbb{N}(\gamma) ; x \rightarrow q_{i}^{*}\left(\omega(x), \omega^{\prime}(x)\right)$ is obviously $d i_{1}$-integrable on $\left\{x_{i}\right\}$, for all $\omega, \omega^{\prime}$ in $\mathcal{D}\left(T^{*} X\right)$. Identifying $\mathcal{D}\left(T^{*} X\right)_{\left\{x_{i}\right\}}$ with $T_{x_{i}}^{*} X$, it follows that $\varepsilon_{i}$ is an energy machinery, the scalar product being given on $\mathcal{D}\left(T^{*} X\right)_{\left\{x_{i}\right\}}$ by $\left(\omega\left(x_{i}\right), \omega^{\prime}\left(x_{i}\right)\right)_{\epsilon_{i}}=\rho\left(x_{i}\right) q_{i}^{*}\left(\omega\left(x_{i}\right), \omega^{\prime}\left(x_{i}\right)\right)$, which, for a given pair $\left(\omega, \omega^{\prime}\right)$ of compactly supported 1-forms, equals to zero outside a finite subset of $\mathrm{N}(\gamma)$; it follows that $i \rightarrow\left(\omega\left(x_{i}\right), \omega^{t}\left(x_{i}\right)\right)_{\varepsilon_{i}}$ is $d n_{\gamma}$-integrable and:

$$
\int_{N(\gamma)}\left(\omega\left(x_{i}^{\prime}, \omega^{\prime}\left(x_{i}\right)\right)_{\varepsilon_{i}} d n_{\gamma}(i)=\sum_{x_{i} \in \gamma} \rho\left(x_{i}\right) q_{i}^{*}\left(\omega\left(x_{i}\right), \omega^{\prime}\left(x_{i}\right)\right)\right. \text {. }
$$

c) In the following example we suppose that $\operatorname{dim}(X) \geq 2$. Let $\overline{\mathbf{N}^{*}}$ be the set $\mathbf{N}^{*} \cup\{\infty\}$; if $k$ is an element of $\mathbf{N}^{*},[k]$ will be the set $\{1,2, \ldots, k\}$; of course, $[\infty]=\mathbb{N}^{*}$.

Definition 5. Let $F$ be a smooth subbundie of $T X$. A family $\tilde{F}$ of connected submanifolds of $X$ will be said subordinate to $F$ if:

(1) There exists $k$ in $\overline{\mathbb{N}^{*}}$ such that $\tilde{F}=\left(Y_{i}\right)_{i \in[k]}$, and for all $i$ in $[k]$ the restricted bundle $F^{Y_{i}}=T Y_{i}$;

(2) $Y_{i} \cap Y_{j}=\phi$ for all $i, j$ in $[k]$ such that $i \neq j$;

(3) for any compact subset $K$ of $X$ there exists a finite subset $\tilde{K}$ of $[k]$ such that $Y_{i} \cap K=\phi$ for all $i$ in $[k]-\tilde{K}$.

\section{Remarks:}

1) All the submanifolds of a family $\widetilde{F}$ subordinate to $F$ have the same dimension $\operatorname{dim}(F)$.

2) Let $F$ be a smooth subbundle of $T X$; any finite family of connected submanifolds of $X$ satisfying properties (1) and (2) of the above definition is subordinate to $F$. 
3) Let $F$ be an integrable smooth subbundle of $T X$; any almost countable farnily of leaves of the foliation coming from $F$ (see e.g. [5], [13]) and satisfying (3) is subordinate to $F$.

Let $\tilde{F}=\left(Y_{i}\right)_{i \in[k]}$ be a family of connected submanifolds of $X$ subordinate to some subbundle $F$ of $T X$, and let $\tau$ be a Riemannian structure on $X$; $\tau$ induces on each submanifold $Y_{i}, i$ in $[k]$, a Riemannian structure $\tau^{i}$, together with a volume measure $d v^{i}$ and an Euclidean structure $q^{r^{i}}$ on $T Y_{i}$; it follows that for all families $\left(\rho_{i}\right)_{i \in[k]}$ such that $\rho_{i}$ is in $C_{+}^{1}\left(Y_{i}\right)$, for all $i$ in $[k]$, the quadruplet $\varepsilon_{i}^{\tau}=\left(Y_{i}, \rho_{i} d v^{i}, F, q^{\tau^{i}}\right)$ is an energy machinery.

Let $d n_{[k]}$ be the counting measure on $[k]$.

Let $\omega, \omega^{\prime}$ be in $\mathcal{D}\left(T^{*} X\right)$; as $\omega$ and $\omega^{\prime}$ are compactly supported $\left(\omega_{Y_{i}}^{F}, \omega_{Y_{i}^{\prime}}^{\prime F}\right)_{\varepsilon_{i}^{r}}=$ $\int_{Y_{i}}\left(q_{x}^{\tau^{i}}\right)^{*}\left(\omega^{F}(x), \omega^{\prime F}(x)\right) \rho_{i}(x) d v^{i}(x)$ is zero except for a finite subset of $[k]$; it follows that $i \rightarrow\left(\omega_{Y_{i}}^{F}, \omega_{Y_{i}}^{\prime F}\right)_{\varepsilon_{i}^{\tau}}$ is $d n_{[k]}$-integrable.

From the above study it follows that Lemma 6 holds:

Lemma 6. Let $F$ be a smooth subbundle of $T X$, let $\tau$ be a Riemannian structure on $X$, let $\tilde{F}=\left(Y_{i}\right)_{i \in[k]}$ be a family of connected submanifolds of $X$ subordinated to $F$, where $k$ is some element of $\overline{\mathrm{N}^{*}}$, and let $\left(\rho_{i}\right)_{i \in[k]}$ be a family such that for all $i$ in $[k], \rho_{i}$ is an element of $C_{+}^{1}\left(Y_{i}\right)$. The assignment $i \rightarrow$ $\varepsilon_{i}^{\tau}=\left(Y_{i}, \rho_{i} d v^{i}, F, q^{\tau^{i}}\right)$, is a $\left([k], d n_{[k]}\right)$-measurable field of energy machineries of type $F$.

Definition 6. The fields of energy machineries of the type given in Lemma 6 are called $(F,[k], \tau)$-fields of energy machineries. When the codimension of $F$ is 1 , they are called maximal $(F,[k], r)$-fields.

\section{5. $(\tau, \mathcal{H}, F, \Lambda, d \ell)$-Energy Structures}

Definition 7. Let $X$ be endowed with the Riemannian structure $\tau$, let $\mathcal{H}$ be a non zero smooth subbundle of $T X$, let $F$ be a smooth subbundle of $\mathcal{H}$, and let $(\Lambda, d \ell)$ be a standard Borel measure space. A $(\tau, \mathcal{H}, F, \Lambda, d \ell)$-energy structure on $X$ is a family $E=\left(E_{p}, E_{c}\right)$ of energy machineries such that:

(1) $E_{p}=\left(X, r d v^{\tau}, \mathcal{H}, q\right)$ is a complete $\tau$-energy machinery,

(2) $E_{\mathrm{c}}=\left(\varepsilon_{\alpha}\right)_{\alpha \in \Lambda}$, with $\varepsilon_{\alpha}=\left(Y_{\alpha}, d \nu_{\alpha}, F, q_{\alpha}\right)$, is a $(\Lambda, d \ell)$-measurable field of energy machineries of type $F$.

$E_{p}$ and $E_{c}$ are respectively called the principal part and the complementary part of $E$.

Lemma 7. Let $E$ be $a(\tau, \mathcal{H}, F, \Lambda, d \ell)$-energy structure on $X: E=\left(E_{p}, E_{c}=\right.$ $\left.\left(Y_{\alpha}, d \nu_{\alpha}, F, q_{\alpha}\right)_{\alpha \in \Lambda}\right)$. The mapping:

$$
\left(\omega^{\mathcal{H}}, \omega^{\prime \mathcal{H}}\right) \longrightarrow\left(\omega^{\mathcal{H}}, \omega^{\prime \mathcal{H}}\right)_{E}=\left(\omega^{\mathcal{H}}, \omega^{\prime \mathcal{H}}\right)_{E_{p}}+\int_{\Lambda}\left(\omega_{y_{\alpha}}^{F}, \omega_{Y_{\alpha}}^{F}\right)_{\varepsilon_{\alpha}} d \ell(\alpha)
$$


is a positive definite inner product on $\mathcal{D}\left(T^{*} X\right)^{\mathcal{H}}$.

Proof: Let $E_{p}=\left(X, r d v^{\top}, \mathcal{H}, q\right)$ be the principal part of $E$; from Lemma 1 it follows that:

$$
\left(\omega^{\mathcal{H}}, \omega^{i \mathcal{H}}\right) \longrightarrow\left(\omega^{\mathcal{H}}, \omega^{\prime \mathcal{H}}\right)_{E_{p}}=\int_{X} q_{x}^{*}\left(\omega^{\mathcal{H}}(x), \omega^{\prime \mathcal{H}}(x)\right) r(x) d v^{\tau}(x)
$$

is a positive definite inner product on $\mathcal{D}(T X)^{F}$. Moreover, for all $\omega$ in $\mathcal{D}\left(T^{*} X\right)$, since $F \subset \mathcal{H}$, the restriction of $\omega^{\mathcal{H}}$ to $F$ is exactly $\omega^{F}$; it follows from Definition 4 that $\left(\omega_{X(\Lambda)}^{F}, \omega_{X(\Lambda)}^{\prime F}\right) \rightarrow \int_{\Lambda}\left(\omega_{Y_{\alpha}}^{F}, \omega_{Y_{\alpha}}^{\prime F}\right)_{\varepsilon_{\alpha}} d \ell(\alpha)$ is a positive definite inner product on $(T X)_{X(\Lambda)}^{F}$, from which it follows that: $\left(\omega^{H}, \omega^{\prime H}\right) \rightarrow \int_{\Lambda}\left(\frac{F}{Y} \omega, \omega_{Y_{\alpha}}^{t F}\right)_{\varepsilon_{\alpha}} d \ell(\alpha)$ is a positive inner product on the space of 1-forms $\mathcal{D}\left(T^{*} X\right)^{\mathcal{H}} .(,)_{E}$ is then the sum of a positive definite inner product and of a positive inner product, hence is a positive definite inner product on $\mathcal{D}\left(T^{*} X\right)^{\mathcal{H}}$.

Remark: When $E=\left(E_{p}, E_{c}\right)$ is a $(\tau, \mathcal{H},(0) \Lambda, d \ell)$-energy structure, then $(,)_{E}=(, \quad)_{E_{p}}$. This fact allows to identify a $(\Lambda, d \ell)$-measurable feld of energy machineries of type (0) with an object that we shall call the vacuum evergy machinery and that we shall denote $\phi$. It follows that energy structures of the form $E=\left(E_{p}, \phi\right)$ will be identified with the complete $\tau$-energy machineries: $E=E_{p}=\left(X, r d v^{r}, \mathcal{H}, q\right)$.

\section{Definition 8.}

(1) The energy structures of the form: $E=\left(X, r d v^{\top}, \mathcal{H}, q\right)$ are called the simple $r$-energy structures.

(2) Among the simple $\tau$ - energy structures the ones of the form $E=\left(X, r d v^{r}\right.$, $T X, q)$ are called the principal $\tau$-energy structures.

(3) Among the principal $\tau^{\prime}$-energy structures the ones of the form $E=$ $\left(X, r d v^{\tau}, T X, q^{\tau}\right)$ are called the basic $\tau$-energy structures.

(4) The energy structures of the form $E=\left(E_{p}, E_{c}\right)$ with $E_{p}$ a basic $\tau$ energy structure, and with $E_{c}$ a $\left(F,[k], \tau^{\prime}\right)$-field of energy machineries are called $\left(\tau, F,[k], \tau^{\prime}\right)$-energy structures; if moreover the codimension of $F$ is $1, E$ is called a maximal $\left(\tau, F,[k], \tau^{\prime}\right)$-energy structure.

As a corollary of Lemma 7 one gets:

Lemma 8. Let $E=\left(E_{p}, E_{c}\right)$ be an energy structure whose principal part $E_{p}$ is a principal $\tau$-energy structure; $(,)_{E}$ is a positive definite inner product on $\mathcal{D}\left(T^{*} X\right)$.

\section{The Orthogonal Representation $V^{E}$ of a Gauge Group}

a) Let $X$ be a smooth connected Riemannian manifold and let $G$ be a compact semisimple Lie group with Lie algebra $g$ endowed with its canonical scalar product $<$. > given by the opposite of its Killing form (which is invariant 
by the adjoint representation $A d$ of $G$ into $g$ ). In accordance with the practice in quantum field and gauge field theories the nuclear Lie group $\mathcal{D}(X, G)$ of all the $G$-valued compactly supported smooth mappings on $X$ will be called a gauge group (see e.g. [2]); its Lie algebra is the space $\mathcal{D}(X, g)$ of $g$-valued compactly supported smooth mappings on $X$. For any subspace $\mathcal{V}$ of $g, \mathcal{D}(X, V)$ will be the nuclear space of $\mathcal{V}$-valued compactly supported smooth mappings on $X$, and $\mathcal{D}_{1}(X, V)$ will be the nuclear space of $\mathcal{V}$-valued compactly supported smooth 1 -forms on $X$. If $F$ is a smooth subbundle of $T X, \mathcal{D}_{1}(X, \mathcal{V})^{F}$ will be the space of restrictions $\omega^{F}$ to $F$ of the elements $\omega$ of $\mathcal{D}_{1}(X, \mathcal{V})$.

We have of course the following equalities:

$$
\left.\begin{array}{l}
\mathcal{D}_{1}(X, \mathcal{V})=\mathcal{D}\left(T^{*} X\right) \otimes \mathcal{V} \\
\mathcal{D}_{1}(X, \mathcal{V})^{F}=\mathcal{D}\left(T^{*} X\right)^{F} \otimes \mathcal{V}
\end{array}\right\}
$$

b) Let $E$ be a $(\tau, \mathcal{H}, F, \Lambda, d \ell)$-energy structure on $X$ and let $\mathcal{V} \neq(0)$ be a subspace of $g$. It follows from Lemma 7 that we can endow $\mathcal{D}_{1}(X, \mathcal{V})^{\mathcal{H}}$ with a positive definite inner product $<,>_{E}$ such that for all elements $\omega^{\mathcal{H}} \otimes u, \omega^{\prime \mathcal{H}} \otimes u^{\prime}$ in the space $\mathcal{D}(T X)^{\mathcal{H}} \otimes \mathcal{V}=\mathcal{D}_{1}(X, \mathcal{V})^{\mathcal{H}}$ :

$$
<\omega^{\mathcal{H}} \otimes u, \omega^{\mathcal{H}} \otimes u^{\prime}>_{E}=\left(\omega^{\mathcal{H}}, \omega^{\prime \mathcal{H}}\right)_{E}<u, u^{\prime}>.
$$

For all $\breve{g}$ in $\mathcal{D}(X, G)$ let us consider the operator $V^{E}(\bar{g})$ on the real prehilbertian space:

$$
\left(\mathcal{D}_{1}(X, g)^{\mathcal{H}},<,>_{E}\right)
$$

such that, for all $\omega^{\mathcal{H}}$ in $\mathcal{D}_{1}(X, g)^{\mathcal{H}}, V^{E}(\bar{g}) \omega^{\mathcal{H}}$ is the 1-form:

$$
x \longrightarrow\left(V^{F_{1}}(\bar{g}) \omega^{\mathcal{H}}\right)(x)=\operatorname{Ad} \bar{g}(x) \cdot \omega^{\mathcal{H}}(x) .
$$

As $G$ acts unitarily on $g$ (with respect to $<, \quad>$ ) by its adjoint representation $A d$, it follows that:

Lemma 9. Let $E$ be a $(\tau, \mathcal{H}, F, \Lambda, d \Omega)$-energy structure on $X$; the assignment $\bar{g} \rightarrow V^{E}(\bar{g})$ is an orthogonal representation of $\mathcal{D}(X, G)$ into $\left(\mathcal{D}_{1}(X, g)^{\mathcal{H}}\right.$, $<,>_{E}$ ). Of course the continuity of Ad on $G$ implies the continuity of $V^{E}$ on $\mathcal{D}(X, G)$; consequently we can extend $V^{E}$ in the following two ways:

i) Firstly, we extend $V^{E}$ into a continuous unitary representation of $\mathcal{D}(X, G)$ into the complex Hilbert space $h^{E}(g)$ generated by $\mathcal{D}_{1}(X, g)^{H}$ with respect to $<,>_{E ;}$;

ii) Secondly, we extend $V^{E}$ by transposition into a continuous representation of $\mathcal{D}(X, G)$ into the dual space $\mathcal{D}_{1}^{\prime}(X, g)^{\mathcal{H}}$ of the nuclear space $\mathcal{D}_{1}(X, g)^{\mathcal{H}}$; $\left\langle V^{E}(\bar{g}) \xi, \omega^{\mathcal{H}}\right\rangle=\left\langle\chi, V^{E}\left(\hat{g}^{-1}\right) \omega^{\mathcal{H}}\right\rangle$, for all $\bar{g}$ in $\mathcal{D}(X, G)$, all $\chi$ in $\mathcal{D}_{1}^{\prime}(X, g)^{\mathcal{H}}$, and all $\omega^{\mathcal{H}}$ in $\mathcal{D}_{1}(X, g)^{\mathcal{H}}$. 
These extensions remain denoted by $V^{E}$.

c) Let us consider now the so-called Maurer-Cartan cocycle $b: \mathcal{D}(X, G) \rightarrow$ $\mathcal{D}_{1}(X, g)$, given for all $\bar{g}$ in $\mathcal{D}(X, G)$ by:

$$
b(\bar{g})=d \bar{g} \cdot \bar{g}^{-1}
$$

It is well-known that for each $x$ in $X$ and all $\bar{g}, \bar{g}^{\prime}$ in $\mathcal{D}(X, G)$ one has:

$$
b\left(\bar{g} \bar{g}^{\prime}\right)(x)=b(\vec{g})(x)+A d \bar{g}(x) \cdot b\left(\bar{g}^{\prime}\right)(x) .
$$

Let $E$ be a $(\tau, \mathcal{H}, F, \Lambda, d \ell)$-energy structure on $X$, and let $b^{\mathcal{H}}: \mathcal{D}(X, G) \rightarrow$ $\mathcal{D}_{1}(X, g)^{\mathcal{H}}$ the mapping $\bar{g} \rightarrow b^{\mathcal{H}}(\bar{g})=(b(\bar{g}))^{\mathcal{H}}$. From the definition of $V^{E}$ and from (11) it follows that for all $\bar{g}, \bar{g}^{\prime}$ in $\mathcal{D}(X, G)$ :

$$
b^{\mathcal{H}}\left(\bar{g} \bar{g}^{\prime}\right)=b^{\mathcal{H}}(\bar{g})+V^{E}(\bar{g}) b^{\mathcal{H}}\left(\bar{g}^{\prime}\right) .
$$

Let $\left(\bar{g}_{p}\right)_{p}$ be a sequence in $\mathcal{D}(X, G)$ and let $\bar{g}$ be in $\mathcal{D}(X, G)$ such that $\lim _{p \rightarrow+\infty} \bar{g}_{p}=\bar{g}$ with respect, to the Schwartz topology of the nuclear Lie group $\mathcal{D}(X, G)$; the sequence $\left(d \bar{g}_{p}\right)_{p}$ converges, with respect to the Schwartz topology, to the corresponding differential mapping $d \bar{g}$ of $\bar{g}$, and then, with respect to the Schwartz topology of $\mathcal{D}_{1}\left(X^{*}, g\right)^{\mathcal{H}}=\mathcal{D}(T X)^{\mathcal{H}} \otimes g, \lim _{p \rightarrow+\infty} b^{\mathcal{H}}\left(\bar{g}_{p}\right)=b^{\mathcal{H}}(\bar{g})$.

It follows that $b^{\mathcal{H}}$ is a continuous 1 -cocycle of $\mathcal{D}(X, G)$ with respect to $V^{E}$. Moreover, $b^{\mathcal{H}}$ cannot be a 1 -coboundary; for all $\bar{g}$ in $\mathcal{D}(X, G), b^{\mathcal{H}}(\bar{g})$ depends on the first derivative of $\bar{g}$, while, for any element $\omega^{\mathcal{H}}$ of $h^{E}(g)$, the corresponding 1-coboundary $V^{E}(\bar{g}) \omega^{\mathcal{H}}-\omega^{\mathcal{H}}$ depends only on $\bar{g}$; this argument of order in the sense of [10] proves that $b^{\mathcal{H}}$ cannot be a 1 -coboundary, and therefore we have:

Lemma 10. Let $E$ be a $(r, \mathcal{H}, F, \Lambda, d \ell)$-energy structure on $X$, and let $G$ be $a$ compact semisimple Lie group with Lie algebra $g . b^{\mathcal{H}}$ is a continuous non trivial 1 -cocycle of $\mathcal{D}(X, G)$ with respect to the continuous unitary representation $V^{E}$.

\section{The $(\tau, \mathcal{H}, F, \Lambda, d \ell)$-Energy Representations of $\mathcal{D}(X, G)$}

a) Let $\mathcal{D}(X, G)$ be a gauge group, and let $E$ be a $(r, \mathcal{H}, F, \Lambda, d \ell)$-energy structure on $X$; we shall denote by $S h^{E}(g)$ the symmetric Hilbert space based on the complex Hilbert space $h^{E}(g)$ generated by $\mathcal{D}_{1}(X, g)^{\mathcal{H}}$ with respect to <

$>_{E}$. Taking into account the Lemma 10 , the general procedure described in $[6]$ yields a unitary representation $\cup^{E}$ of type $(S)$ of $\mathcal{D}(X, G)$ into $S h^{E}(g)$ such that, on the total set $\operatorname{EXP(hE}(g))$, for all $\bar{g}$ in $\mathcal{D}(X, G)$, and for all $\omega^{\mathcal{H}}$ in $h^{E}(g)$ (the notations being the ones used in [16]):

$$
\begin{aligned}
& U^{E}(\bar{g}) E X P \omega^{\mathcal{K}}=\exp \left\{-\frac{1}{2}\left\|b^{\mathcal{H}}(\bar{g})\right\|_{E}^{2}-<V^{E}(\bar{g}) \omega, b^{\mathcal{H}}(\bar{g})>_{E}\right\} \\
& \operatorname{EXP}\left(V^{E}(\bar{g})+b^{\mathcal{H}}(\bar{g})\right) \text {. }
\end{aligned}
$$

One easily sees that such a representation is of order 1 and its support is the whole manifold $X$. If follow that the following theorem holds: 
Theorem 1. To each $(\tau, \mathcal{H}, F, \Lambda$, de)-energy structure $E$ on the manifold $X$ there is a continuous unitary representation $\cup^{E}$, given by (13), of the gauge group $\mathcal{D}(X, G)$, with support $X$ and order 1 . The corresponding spherical func. tion $\varphi_{E}: \bar{g} \rightarrow<\cup^{E}(\bar{g}) E X P O, E X P O>$ with respect to the vacuum vector $E X P O$ is given by $\varphi_{E}(\vec{g})=\exp \left\{-\frac{1}{2}\left\|b^{\mathcal{H}}(\bar{g})\right\|_{E}^{2}\right\}$.

Definition 9: Let $E$ be a $(\tau, \mathcal{H}, F, \Lambda, d \ell)$-energy structure on $X$; the corresponding representation $\sqcup^{E}$ of $\mathcal{D}(X, G)$ is called a $(\tau, \mathcal{H}, F, \Lambda$, dl)-energy representation.

b) Historical Note. The first energy representation $\cup^{E}$ was given by $R$. Ismagilov in [7], with $X$ an open subset of $\mathrm{R}^{n}, G=S U(2)$, and $E=$ $\left(X, d x, X \times \mathbb{R}^{n}, q_{0}\right)$, where $d x$ is the Lebesgue measure on $X, q_{0}$ being the canonical Euclidean structure on $\mathbb{R}^{n}$. A series of papers of A.M. Vershik, I.M. Gelfand and M.I. Graev ([16], [17], [18]) followed; this first work gave, for any gauge group $\mathcal{D}(X, G)$, the energy representations $\cup^{E}, E$ being a simple $\tau-$ energy structure of the type $\left(X, d v^{\tau}, \mathcal{H}, q_{\tau}^{\mathcal{H}}\right)$. In [1] S. Albeverio and R. HøeghKrohn gave another realization of the same $\cup^{E}$; it is in this paper that, for the first time, appeared the expression energy representation, which comes from the fact that the corresponding spherical function $\varphi_{E}$ can be looked at as a kind of integral of energy. Then S. Albeverio, R. Høegh-Krohn and D. Testard studied energy representations $\sqcup^{E}$ with $E=\left(X, \rho d v^{\tau}, T X, q_{\tau}\right), \rho$ in $C_{+}^{1}(X) \cap C^{\infty}(X)$, in [2]. In the case of a manifold $X$ with Euler number $e(X)=0 \mathrm{~J}$. Marion, in [9], gave energy representations $\cup^{E}$ with $E=\left(X, \tau d v^{\tau}, F(\xi), q_{\xi}\right)$ of the type described in Lemma 3 , with $r$ in $C_{+}^{l}(X)$. A survey of these various $\sqcup^{E}, E$ being always a simple $\tau$-energy machinery is given in [11]; in this paper was raised the question of the existence of other types of energy representations; a partial answer to this question is given in the present work.

c) Let us give another useful and convenient realization of the representation $\sqcup^{E}$. The spherical function $\varphi_{E}$ of $U^{E}$ is a continuous function of definite positive type on $\mathcal{D}(X, G)$; it induces a positive definite function $\tilde{\varphi}_{E}$ on the real nuclear space $\mathcal{D}_{1}(X, g)^{\mathcal{H}}$, given by:

$$
\varphi_{E}\left(\omega^{\mathcal{H}}\right)=e^{\left.-\frac{1}{2} \mid\right] \omega^{\mathcal{H}} \|_{E}^{2}}, \omega^{F} \in \mathcal{D}_{1}(X, g)^{\mathcal{H}} .
$$

Now let us recall that $\mathcal{D}_{1}(X, g)^{\mathcal{H}}$ is a real nuclear space, inductive limit of Fréchet spaces; it follows then from the Bochner-Minloss theorem that there exists a unique gaussian measure $\mu_{E}$ on the dual space $\mathcal{D}_{1}^{\prime}(X, g)^{\mathcal{H}}$ whose Fourier transforn $\hat{\mu}_{E}$ is given by:

$$
\hat{\mu}_{E}\left(\omega^{\mathcal{K}}\right)=\tilde{\varphi}_{E}\left(\omega^{\mathcal{H}}\right)=e^{t}-\frac{1}{2}\left\|\omega^{\mathcal{H}}\right\|_{E}^{2}, \omega^{F} \in \mathcal{D}_{1}(X, g)^{\mathcal{H}}
$$

The theorem 7.9 of [6] allows then the realization of $\sqcup^{E}$ in the Hilbert space $L^{2}\left(\mathcal{D}_{1}^{\prime}(X ; g)^{\mathcal{H}} ; \mu_{E}\right)$; in this picture $\cup^{E}$ is given by:

$$
\sqcup^{E}(\bar{g}) \Phi(\chi)=\exp \left\{i<b^{\mathcal{H}}(\bar{g}), \chi>\right\} \cdot \Phi\left(V^{E}\left(\bar{g}^{-1}\right) \chi\right),
$$


$V^{E}$ being here the representation of $\mathcal{D}(X, G)$ extended by transposition into $\mathcal{D}_{1}^{\prime}(X, G)^{\mathcal{H}}$ (see $\S$ III.6,b), for all $\bar{g}$ in $\mathcal{D}(X, G), \Phi$ in $L^{2}\left(\mathcal{D}_{1}^{\prime}(X, g)^{\mathcal{H}} ; \mu_{E}\right), \chi$ in $\mathcal{D}_{1}^{\prime}(X, g)^{\mathcal{H}}$.

d) The main question is row to recognize what are the energy representations which are irreducible (if so, their classe are $G$-distributions of order 1 and with support $X$ ), or, at least, what are the ones which are cyclic. The following lemma shows that if suffices to know the answer in the case of energy representations $\sqcup^{E}, E$ being a $(\tau, T X, F, \Lambda, d \ell)$-energy structure, i.e. of the form $E=\left(E_{p}, E_{c}\right)$ with $E_{p}$ a principal $\tau$-energy structure $\left(X, r d v^{\tau}, T X, q\right)$.

Let $E=\left(\left(X, r d v^{\tau}, \mathcal{H}, q\right), E_{c}\right)$ be a $(\tau, \mathcal{H}, F, \Lambda, d \ell)$-energy structure with principal part $E_{p}=\left(X, r d v^{\tau}, \mathcal{H}, q\right), H \neq T X$, let $q_{\tau}^{\perp}$ be the restriction of the Euclidean structure $q_{\tau}$ coming from $\tau$ to the orthogonal subbundle $\mathcal{H}^{\perp}$ of $\mathcal{H}$ with respect to this Riemannian structure. $E^{r}=\left(X, r d v^{\tau}, \mathcal{H}^{\perp}, q_{\tau}^{\perp}\right)$ is an energy machinery; moreover, let $q \oplus q_{\tau}^{\perp}$ be the Euclidean structure on $T X=\mathcal{H} \oplus \mathcal{H}^{\perp}$ such that $q \oplus q_{\tau}^{\perp}$ restricted to $\mathcal{H}$ equals $q$, and such that $q \oplus q_{\tau}^{\perp}$ restricted to $\mathcal{H}^{\perp}$ equals $q_{\tau}^{\perp}$. From Lemma 4 it follows that:

$$
E_{p} * E^{r}=\left(X, r d v^{\tau}, T X, q \oplus p_{\tau}^{\perp}\right)
$$

is a regular complete $\tau$-energy machinery. As an obvious consequence it follows that $E * E^{r}=\left(E_{p} * E^{\tau}, E_{c}\right)$ is a $(\tau, T X, F, \Lambda, d \ell)$-energy structure on $X$. In accordance with the definition given in Lemma $4, E * E^{\tau}$ will be called the $\tau \sim$ regularization of $E$.

Lemma 11. Let $E$ be a $(\tau, \mathcal{H}, F, \Lambda, d \ell)$-energy structure with $\mathcal{H} \neq T X$, and let $E * E^{r}$ be its $\tau$-regularization.

$$
\sqcup^{E \star E^{\gamma}} \text { is unitarily equivalent to } \sqcup^{E} \oplus \mathrm{U}^{E^{r}} \text {. }
$$

Proof: In the proof of Lemma 4 the orthogonal decomposition with respect to $\tau$ was shown

$$
\mathcal{D}\left(T^{*} X\right)=\mathcal{D}\left(T^{*} X\right)^{\mathcal{H}} \oplus \mathcal{D}\left(T^{*} X\right)^{\mathcal{H}^{\perp}} .
$$

This orthogonal decomposition remains true with $\mathcal{D}\left(T^{*} X\right)$ endowed with the scalar product $(,)_{E * E^{*}}$ which is the sum of the scalar product $(,)_{E}$ on $\mathcal{D}\left(T^{*} X\right)^{\mathcal{H}}$ and of the scalar product $(,)_{E}$ on $\mathcal{D}\left(T^{*} X\right)^{\mathcal{H}^{\perp}}$.

It follows the orthogonal decomposition:

$$
\mathcal{D}_{1}(X, g)=\mathcal{D}_{1}(X, g)^{\mathcal{H}} \oplus \mathcal{D}_{1}(X, g)^{\mathcal{H}^{\perp}}
$$

with respect to the scalar product $<,,>_{E * E^{r}}$ which is the sum of $<,>_{E}$ on $\mathcal{D}_{1}(X, g)^{\mathcal{H}}$ and of $<, \quad>_{E^{r}}$ on $\mathcal{D}_{1}(X, g)^{\mathcal{H}^{\perp}}$.

Let $\mu_{E}, \mu_{E^{+}}$and $\mu_{E^{*} E^{r}}$ be the Gaussian measures on respectively $\mathcal{D}_{1}^{\prime}(X, g)^{\mathcal{H}}$, $\mathcal{D}_{1}^{\prime}(X, g)^{\mathcal{H}^{\perp}}$ and $\mathcal{D}_{1}^{\prime}(X, g)$ with corresponding Fourier transforms given by:

$$
\begin{aligned}
& \hat{\mu}_{E}\left(\omega^{\mathcal{H}}\right)=\exp \left\{-\frac{1}{2}\left\|\omega^{\mathcal{H}}\right\|_{E}^{2}\right\} \quad, \omega^{\mathcal{H}} \quad \text { in } \mathcal{D}_{1}(X, g)^{\mathcal{H}} \text {, } \\
& \hat{\mu}_{E^{r}}\left(\omega^{\mathcal{H}^{\perp}}\right)=\exp \left\{-\frac{1}{2}\left\|\omega^{\mathcal{H}}\right\|_{E^{r}}^{2}\right\}, \omega^{\mathcal{H}^{\perp}} \text { in } \mathcal{D}_{1}(X, g)^{\mathcal{H}^{\perp}} \text {, } \\
& \hat{\mu}_{E^{*} E^{r}}(\omega)=\exp \left\{-\frac{1}{2}\|\omega\|_{E^{*} E^{r}}^{2}\right\}, \omega \quad \text { in } \mathcal{D}(X, g) \text {. }
\end{aligned}
$$


From $\left\|\omega_{E^{*} E^{\tau}}^{2}=\right\| \omega^{\mathcal{H}}\left\|_{E}^{2}+\right\| \omega^{\mathcal{H}^{1}} \|_{E^{*}}$, it follows that:

$$
\mu_{E^{*} E^{r}}=\mu_{E} \oplus \mu_{E},
$$

and then, taking account (17), one gets:

$$
L^{2}\left(\mathcal{D}_{1}^{\prime}(X, g) ; \mu_{E^{\cdot} E^{r}}\right) \simeq L^{2}\left(\mathcal{D}_{1}^{\prime}(X, g)^{\mathcal{H}} ; \mu_{e}\right) \otimes L^{2}\left({ }_{1}^{\prime}(X, g)^{\mathcal{H}^{\perp}} ; \mu_{E^{r}}\right) .
$$

It follows then from (16) that $\sqcup^{E^{*} E^{r}}=\sqcup^{E} \otimes \sqcup^{E^{r}}$.

Corollary. If $\cup^{E^{*} E^{r}}$ is irreducible, $\cup^{E}$ is irreducible, too.

\section{The Unitary Representation $\Pi_{\mathcal{A}}^{E}$ and its Spectral Measure}

Let $E$ be a $(\tau, T X, F, \Lambda, d \ell)$-energy structure on $X$, let $\sqcup^{E}$ be the corresponding energy representation of the gauge group $\mathcal{D}(X, G)$, and let $\mathcal{A}$ be a Cartan subalgebra of $g$; the orthogonal complement of $\mathcal{A}$ in $g$ with respect to the canonical scalar product $<,>$ on $g$ will be denoted by $\mathcal{A}^{\perp}$, and the maximal torus $\exp (\mathcal{A})$ in $G$ will be denoted by $T$.

We introduce here the spaces $\mathcal{D}_{1}(X, \mathcal{A})=\mathcal{D}\left(T^{*} X\right) \otimes \mathcal{A}, \mathcal{D}_{1}\left(X, \mathcal{A}^{\perp}\right)=$ $\mathcal{D}\left(T^{*} X\right) \otimes \mathcal{A}^{\perp} ;$ from the orthogonal sum:

$$
g=\mathcal{A} \oplus \mathcal{A}^{\perp} \text { (with respect to }<, \quad>\text { ) }
$$

it follows the orthogonal decomposition with respect to $<,>_{E}$ :

$$
\mathcal{D}_{1}(X, g)=\mathcal{D}_{1}(X, \mathcal{A}) \oplus \mathcal{D}_{1}\left(X, \mathcal{A}^{\perp}\right) .
$$

Of course the Lie algebra of the abelian nuclear Lie group $\mathcal{D}(X, T)$ is the abelian Lic algebra $\mathcal{D}(X, \mathcal{A})$. The energy representation $\sqcup^{E}$ defines a unitary representation $\cup_{\mathcal{A}}^{E}$ of the abelian nuclear group $\mathcal{D}(X, \mathcal{A})$ into the Hibert space $L^{2}\left(\mathcal{D}_{1}^{\prime}(X, g) ; \mu_{E}\right)$ given by:

$$
\Pi_{\mathcal{A}}^{E}(u)=\sqcup^{E}(\exp u), u \in \mathcal{D}(X, \mathcal{A}) .
$$

The present section is devoted to the study of $\Pi_{\mathcal{A}}^{E}$.

a) Let $d: \mathcal{D}(X, \mathcal{A}) \rightarrow \mathcal{D}_{1}(X, \mathcal{A})$ be the exterior derivative, given by $u \rightarrow d u ; d$ is continuous with respect to the Schwartz topologies of $\mathcal{D}(X, \mathcal{A})$ and $\mathcal{D}_{1}(X, \mathcal{A})$ and ker $d$, the space of constant functions in $\mathcal{D}(X, \mathcal{A})$ is a closed subspace which equals $(0)$ if $X$ is a non compact manifold; we shall denote by $\tilde{D}(X, \mathcal{A})$ the space $\mathcal{D}(X, \mathcal{A}) /$ ker $d$, and for any element $u$ in $\mathcal{D}(X, \mathcal{A})$ by $\tilde{u}$ its class in $\tilde{\mathcal{D}}(X, \mathcal{A}), \tilde{\mathcal{D}}(X, \mathcal{A})$ is a nuclear space and $\tilde{d}: \tilde{\mathcal{D}}(X, \mathcal{A}) \rightarrow \mathcal{D}_{1}(X, \mathcal{A})$ such that $\tilde{d}(\tilde{u})=d u$ is a one-to-one continuous linear mapping which allows to endow $\tilde{D}(X, \mathcal{A})$ with the positive definite inner product $\widetilde{E}$ given by;

$$
\tilde{E}(\tilde{u}, \tilde{v})=<\tilde{d} \tilde{u}, \tilde{d} \tilde{v}>_{E}=<d u, d v>_{E} .
$$

It follows that the following lemma holds: 
Lemma 12. $\tilde{d}$ is an isometry with close range of the real prehilbertian space $(\tilde{\mathcal{D}}(X, \mathcal{A}), \tilde{E})$ into the prehilbertian space $\left(\mathcal{D}(X, \mathcal{A}),<, \quad>_{E}\right)$.

Let $\tilde{\mu}_{E}$ be the Gaussian measure on the dual space $\tilde{\mathcal{D}}^{\prime}(X, \mathcal{A})$ with Fourier transformation $\hat{\mu}_{E}: \tilde{u} \rightarrow \dot{\tilde{\mu}}_{E}(\tilde{u})=\exp \left\{-\frac{1}{2} \tilde{E}(\tilde{u}, \tilde{u})\right\}$, let $\mu_{E, \mathcal{A}}$ and $\mu_{E, \mathcal{A}^{\perp}}$ be the Gaussian measures on the dual spaces $\mathcal{D}_{1}^{\prime}(X, \mathcal{A})$ and $\mathcal{D}_{1}^{\prime}\left(X, \mathcal{A}^{\perp}\right)$ given by

$$
\begin{aligned}
& \hat{u}_{E, \mathcal{A}}: \omega \longrightarrow \hat{\mu}_{E, \mathcal{A}}(\omega)=\exp \left\{-\frac{1}{2}<\omega, \omega>_{E}\right\}, \omega \in \mathcal{D}_{1}(X, \mathcal{A}), \\
& \hat{\mu}_{E, \mathcal{A}^{\perp}}: \omega \longrightarrow \hat{\mu}_{E, \mathcal{A}^{\perp}}(\omega)=\exp \left\{-\frac{1}{2}<\omega, \omega>_{E}\right\}, \omega \in \mathcal{D}_{1}\left(X, \mathcal{A}^{\perp}\right) .
\end{aligned}
$$

One gets:

Lemma 13. For all $\tilde{u}$ in $\tilde{\mathcal{D}}(X, \mathcal{A})$ let $\tilde{w}(\tilde{u})$ be the operator on $L^{2}\left(\mathcal{D}_{1}^{\prime}(X, \mathcal{A})\right.$; $\mu_{E, A}$ ) given by:

$$
\tilde{w}(\tilde{u}) \Phi(\chi)=\exp \{i<\chi, d u>\} \Phi(\chi) .
$$

(i) $\tilde{w}: \tilde{u} \rightarrow \tilde{w}(\tilde{u})$ is a continuous unitary representation of $\tilde{\mathcal{D}}(X, \mathcal{A})$ into $L^{2}\left(\mathcal{D}_{1}^{\prime}(X, \mathcal{A}) ; \mu_{E, \mathcal{A}}\right)$.

(ii) The spectral measure of $\tilde{w}$ is equivalent to $\tilde{\mu}_{E}$.

\section{Proof:}

(i) follows from an easy verification.

(ii): Let $\tilde{d}^{*}$ be the transposed mapping of $\tilde{d}$; from Lemma $12 \tilde{d}^{*}$ maps $\mathcal{D}_{1}^{\prime}(X, \mathcal{A})$ onto $\left(\tilde{\mathcal{D}}^{\prime}(X, \mathcal{A})\right.$. As these two spaces are standard Borel spaces there exists a Borel section $s$ of $\tilde{d} *$ such that the mapping:

$$
\tilde{s}: \chi \longrightarrow\left(\tilde{d}^{*} \chi, s \tilde{d}^{*} \chi-\chi\right)
$$

is an isomorphism of Borel spaces from $\mathcal{D}_{1}^{\prime}(X, \mathcal{A})$ onto $\tilde{D}^{\prime}(X, \mathcal{A}) \times k e r \tilde{d}^{*}$, from which it follows that $\tilde{s}\left(\mu_{E, \mathcal{A}}\right)=\tilde{d}^{*} \mu_{E, \mathcal{A}} \times \lambda, \lambda$ being some Borel measure on $k \operatorname{cr} \tilde{d}^{*}$. One gets then an isomorphism of Hilbert spaces:

$$
L^{2}\left(\mathcal{D}_{1}^{\prime}(X, \mathcal{A}) ; \mu_{E, A}\right) \simeq L^{2}\left(\tilde{\mathcal{D}}^{\prime}(X, \mathcal{A}) ; \tilde{\mu}_{E}\right) \otimes L^{2}\left(k \operatorname{ker} \tilde{d}^{*} ; \lambda\right)
$$

such that for all $\tilde{u}$ in $\tilde{\mathcal{D}}(X, \mathcal{A}), \tilde{w}(\tilde{u})$ is transformed into the operator $\tilde{w}(\tilde{u}) \otimes$ $\tilde{\coprod}, \tilde{w}^{\prime}$ being the tunitary representation of $\tilde{\mathcal{D}}(X, \mathcal{A})$ into $L^{2}\left(\tilde{\mathcal{D}}^{\prime}(X, \mathcal{A}) ; \tilde{d}^{*} \mu_{E, \mathcal{A}}\right)$ given by:

$$
\tilde{w}^{\prime}(\tilde{u}) \psi(\chi)=\exp \{i<\chi, \tilde{d} \tilde{u}>\} \psi(\chi)
$$

$\psi$ in $L^{2}\left(\tilde{\mathcal{D}}^{\prime}(X, \mathcal{A}) ; \tilde{d}^{*} \mu_{E, \mathcal{A}}\right), \chi \in \tilde{\mathcal{D}}^{\prime}(X, \mathcal{A})$.

It follows that the spectral measure of $\tilde{w}$ is equivalent to $\tilde{d}^{*} \mu_{E, \mathcal{A}}$; as all the spaces interfering here are nuclear spaces, owing to the uniqueness of gaussian measures given by their Fourier transforms, it follows that $\tilde{d}^{*} \mu_{E, \mathcal{A}}$ equals $\tilde{\mu}_{E}$. So, the spectral measure of $\tilde{w}$ is (equivalent to) $\tilde{\mu}_{E}$.

b) Let $\Theta^{E}$ be the unitary representation of $\mathcal{D}(X, \mathcal{A})$ into $L^{2}\left(\mathcal{D}_{1}^{\prime}\left(X, \mathcal{A}^{\perp}\right)\right.$; $\left.\mu_{E, A^{\perp}}\right)$ given by:

$$
\Theta^{E}(u) \Phi(\chi)=\Phi\left(V^{E}(\exp u) \chi\right),
$$

with $\Phi$ in $L^{2}\left(\mathcal{D}_{1}^{\prime}\left(X, \mathcal{A}^{\perp}\right) ; \mu_{E, \mathcal{A}^{\perp}}\right), \chi$ in $\mathcal{D}_{1}^{\prime}\left(X, \mathcal{A}^{\perp}\right)$. 
Lemma 14. The spectral measure $\nu^{E}$ of $\Theta^{E}$ is equivalent to the infinite direct sum:

$$
\bigoplus_{k \geq 0}\left(r d v^{\tau} \otimes N\right)^{\otimes k}
$$

$N$ being the counting measure on the set $\Delta$ of roots of the Cartan pair $(g, \mathcal{A})$.

Proof: We shall use the Fock realization of $L^{2}\left(\mathcal{D}_{1}^{\prime}\left(X, \mathcal{A}^{\perp}\right) ; \mu_{E, \mathcal{A}^{\perp}}\right)$ : let $h^{E}\left(\mathcal{A}^{\perp}\right)$ be the complex Hilbert space spanned by $\mathcal{D}_{1}\left(X, \mathcal{A}^{\perp}\right)$ with respect to $<, \quad>_{E}$; from [6], theorem $7.2, L^{2}\left(\mathcal{D}_{1}^{\prime}\left(X, \mathcal{A}^{\perp}\right) ; \mu_{E, \mathcal{A}^{\perp}}\right)$ is isomorphic to the Fock space

$$
S h^{E}\left(\mathcal{A}^{\perp}\right)=\sum_{p \geq 0}^{\oplus} S^{p} h^{E}\left(\mathcal{A}^{\perp}\right)
$$

$S^{p} h^{E}\left(\mathcal{A}^{\perp}\right)$ being the $p-t h$ symmetric tensor power of $h^{E}\left(\mathcal{A}^{\perp}\right)$. Let $\Delta$ be the set of roots of $g$ with respect to the Cartan subalgebra $\mathcal{A}$, and for $\alpha$ in $\Delta$, let $g^{\alpha}$ be the subspace of $g$ with weight $\alpha$; one gets.

$$
\mathcal{A}^{\perp}=\underset{\alpha \in \Delta}{\oplus} g^{\alpha}
$$

from which it follows that $h^{E}\left(\mathcal{A}^{1}\right)=\underset{\alpha \in \Delta}{\oplus} h^{E}\left(g^{\alpha}\right), h^{E}\left(g^{\alpha}\right)$ being the complex Hilbert space spanned by $\mathcal{D}_{\mathrm{I}}\left(X, g^{\alpha}\right)$ with respect to $<, \quad>_{E}$. It follows that $S h^{E}\left(\mathcal{A}^{\perp}\right)=\underset{\alpha \in \Delta}{\otimes} S h^{E}\left(g^{\alpha}\right)$. For all $\omega$ in $h^{E}\left(g^{\alpha}\right)$, for all $u$ in $\mathcal{D}(X, \mathcal{A})$ one has:

$$
V^{E}(\exp u) \omega: x \longrightarrow e^{i \alpha(u(x))} \omega(x), x \in \chi .
$$

Then the restriction of $\Theta^{E}$ on $S^{p} h^{E}\left(\mathcal{A}^{\perp}\right)$ (which equals $\underset{\alpha \in \Delta}{\otimes} S^{p} h^{E}\left(g^{\alpha}\right)$ ) acts by multiplication by elements of the form $e^{i \sum_{k=1}^{p} \alpha_{k}\left(u\left(x_{k}\right)\right)}$, with $\alpha_{1}, \ldots, \alpha_{p}$ in $\Delta$ and $x_{1}, \ldots, x_{p}$ in $X$. It follows that the spectral measure $\nu^{E}$ of $\Theta^{E}$ is supported by the subset $\Gamma_{\mathcal{A}}$ of $\mathcal{D}^{\prime}(X, \mathcal{A})$ consisting of functionals of the form:

$$
\chi: u \longrightarrow \chi(u)=\sum_{k=1}^{k=p} \alpha_{k}\left(u\left(x_{k}\right)\right)
$$

for all non negative integer $p$, the $\alpha_{k}$ being in $\Delta$ and the $x_{k}$ being in $X$.

We can then identify $\Gamma_{\mathcal{A}}$ with $\bigcup_{p \geq 0}(X \times \Delta)_{\mathrm{sym}}^{p}$, and $\nu^{E}$ with the Poisson measure whose restriction to $(X \times \Delta)_{\mathrm{sym}}^{p}$ is given by $\left(r d v^{r} \otimes n\right)^{\otimes p}, N$ being the counting measure on $\Delta, r d v^{\tau}$ the measure on $X$ given by the principal part of $E$ (see e.g. $[6], \S 3$ ).

c) We come back now to the representation $\Pi_{\mathcal{A}}^{E}$ of $\mathcal{D}(X, \mathcal{A})$ into $L^{2}\left(\mathcal{D}_{1}^{\prime}(X, \mathcal{A})\right.$; $\left.\mu_{E}\right)$ such that, for all $u$ in $\mathcal{D}(X, \mathcal{A})$ :

$$
\Pi_{\mathcal{A}}^{E}(u)=\sqcup^{E}(\exp u)
$$


From (18) it follows that we have the isomorphism:

$$
L^{2}\left(\mathcal{D}_{1}^{\prime}(X, g) ; \mu_{E}\right) \simeq L^{2}\left(\mathcal{D}_{1}^{\prime}(X, \mathcal{A}) ; \mu_{E, \mathcal{A}}\right) \otimes L^{2}\left(\mathcal{D}_{1}^{\prime}\left(X, \mathcal{A}^{\perp}\right) ; \mu_{E, \mathcal{A}^{\perp}}\right)
$$

so, if $u$ is in $\mathcal{D}(X, \mathcal{A})$, and $\Phi=\Phi_{1} \otimes \Phi_{2}$ in the above space, from the definitions of $\cup^{E}, \tilde{w}$ and $\Theta^{E}$, and the fact that for all $u$ in $\mathcal{D}(X, \mathcal{A}), b(\exp u)=d u$, it follows that:

$$
\begin{gathered}
\Pi_{\mathcal{A}}^{E}(u)\left(\Phi_{1} \otimes \Phi_{2}\right)=\left(\tilde{w}(\tilde{u}) \Phi_{1}\right) \otimes\left(\Theta^{E}(u) \Phi_{2}\right), \text { i.e.: } \\
\Pi_{\mathcal{A}}^{E}(u)=\tilde{w}(\tilde{u}) \otimes \Theta^{E}(u) .
\end{gathered}
$$

From Lemma 13 and Lemma 14, one gets then:

Theorem 2. Let $E$ be a $(\tau, T X, F, \Lambda, d \ell)$-energy structure on the manifold $X$, let $\sqcup^{E}$ the corresponding energy representation of the gauge group $\mathcal{D}(X, G)$, let $\mathcal{A}$ be a Cartan subalgebra of $g$, and let $\Pi_{\mathcal{A}}^{E}$ be the unitary representation of the abelian Lie group $\mathcal{D}(X, \mathcal{A})$ such that for all $u$ in $\mathcal{D}(X, \mathcal{A})$;

$$
\Pi_{\mathcal{A}}^{E}(u)=\sqcup^{E}(\exp u)
$$

The spectral measure of $\Pi_{\mathcal{A}}^{E}$ is equivalent to the convolution $\tilde{\mu}_{E} * \nu^{E}, \bar{\mu}_{E}$ being the Gaussian measure on $\tilde{\mathcal{D}}^{\prime}(X, \mathcal{A})$ with Foutier transform $\tilde{u} \rightarrow \tilde{\tilde{\mu}}_{E}(\tilde{u})=$ $\exp \left\{-\frac{1}{2} \hat{i d u} \|_{E}^{2}\right\}$ and with $\nu^{E} \simeq \underset{p \geq 0}{\oplus}\left(r d v^{\tau} \otimes N\right)^{\otimes p}$.

d) We want to give now a direct integral decomposition of $\Pi_{\mathcal{A}}^{E}$.

Let us recall that $\Gamma_{\mathcal{A}}$ is the subset of $\mathcal{D}^{\prime}(X, \mathcal{A})$ of functionals of the form $\chi=\sum_{k \in I \text { finite }}^{\sum} \delta_{x_{k}}^{\alpha_{k}}$, the $\alpha_{k}$ being in $\Delta$, the $x_{k}$ in $X$, and the functional $\delta_{x_{k}}^{\alpha_{k}}$ being given by $\delta_{x_{k}}^{\alpha_{k}}(u)=\alpha_{k}\left(u\left(x_{k}\right)\right), u$ in $\mathcal{D}(X, \mathcal{A})$ (see part b) above).

For each $\chi$ in $\Gamma_{\mathcal{A}}$, let $\gamma_{\chi}$ be the character of $\mathcal{D}(X, \mathcal{A})$ such that $\gamma_{\chi}(u)=$ $\exp (i<\chi, u>), u \in \mathcal{D}(X, \mathcal{A})$, and let $\tilde{w}_{\chi}=\tilde{w} \otimes \gamma_{\chi}$ the unitary representation of $\mathcal{D}(X, \mathcal{A})$, $\tilde{w}$ being the representation defined in Lemma 13. $\tilde{w}_{x}$ can be written also $\tilde{w}_{x}=w \otimes \gamma_{\chi}, w$ being the unitary representation of $\mathcal{D}(X, \mathcal{A})$ defined by:

$$
w(u)=\tilde{w}(\tilde{u}), u \in \mathcal{D}(X, \mathcal{A})
$$

It follows that $w$ and $\tilde{w}$ have the same spectral measure, which is equivalent to $\tilde{\mu}_{E}$ by Lemma 13 , and then the spectral measure $\tilde{\mu}_{E}^{X}$ of $\tilde{w}_{x}$ is the convolution of the spectral measure $\tilde{\mu}_{E}$ of $w$ by the spectral measure of the character $\gamma_{X}$. More precisely:

Lemma 15.

(i) One has the direct integral decomposition:

$$
\Pi_{\mathcal{A}}^{E}=\int_{\Gamma_{\mathcal{A}}}^{\oplus} \tilde{W}_{\chi} d \nu^{E}(\chi)
$$


(ii) $\tilde{\mu}_{E}^{\chi}$ is equivalent to the translated of $\tilde{\mu}_{E}$ by $-\chi$, i.e.:

$$
\tilde{\mu}_{E}^{\chi} \simeq \tilde{\mu}_{E}(\cdot,-\chi) \text {. }
$$

Proof:

(i) From Lemma 14 one gets: $\Theta^{E}=\int_{\Gamma_{\wedge}}^{\oplus} \gamma_{\chi} d \nu \nu^{E}(\chi)$; it follows then from (23) that:

$$
\begin{aligned}
\Pi_{\mathcal{A}}^{E}=\tilde{w} \otimes \Theta^{E} & =\tilde{w} \otimes \int_{\Gamma_{\mathcal{A}}}^{\oplus} d \nu^{E}(\chi)=\int_{\Gamma_{\mathcal{A}}}^{\oplus}\left(\tilde{w} \otimes \gamma_{\chi}\right) d \nu^{E}(\chi) \\
& =\int_{\Gamma_{\mathcal{A}}}^{\oplus} \tilde{w}_{\chi} d \nu^{E}(\chi) .
\end{aligned}
$$

(ii) follows from the fact that the spectral measure of $\tilde{w}_{\chi}$ is the convolution of the spectral measure $\tilde{\mu}_{E}$ of $\tilde{w}$ by the spectral measure of the character $\gamma_{\chi}$ which is given by $\chi$, and them: $\tilde{\mu}_{E}^{\chi}=\tilde{\mu}_{E}(\cdot,-\chi)$.

Remarks:

1) Let $\mathcal{U}$ be the algebra of measurable subsets of $\mathcal{D}^{\prime}(X, \mathcal{A})$; the mapping $(\chi, B) \in \Gamma \times \mathcal{U} \rightarrow \tilde{\mu}_{E}^{\chi}(\chi, B)$ is measurable because $\tilde{\mu}_{E}^{\chi}(\chi, B)=\tilde{\mu}_{E}(B-\chi)$.

2) The results of Lemrnas 13,14,15 and of Theorem 2 are proved in [17] and (2) in the case of a non compact $X$ and of $E$ of the form $\left(X, r d v^{\tau}, T X, q_{\tau}\right)$. The proofs given here in the case of a general $(\tau, T X, F, \Lambda, d \ell)$-energy structure are of the same type; moreover the use of the space $\tilde{\mathcal{D}}(X, \mathcal{A})$ and for $\tilde{d}$, instead of $\mathcal{D}(X, \mathcal{A})$ and $d$, allows to include the case $X$ is compact.

\section{A Sufficient Condition for the Irreducibility of $L^{E}$}

The goal of this section is to prove that under some conditions about uniform disjointness of the Gaussian measures $\tilde{\mu}_{E}^{X}, \sqcup^{E}$ is irreducible.

Definition 10: Let $E$ be a $(\tau, T X, F, \Lambda, d \ell)$-energy structure on $X$, and let $\mathcal{D}(X, G)$ bc a gauge group. We shall say that $\sqcup^{E}$ has the $\Gamma$-property if there exists a Cartan subalgebra $\mathcal{A}$ of $g$ such that for any pair $\left(\Gamma_{1}, \Gamma_{2}\right)$ of subsets of $\Gamma_{\mathcal{A}}$ satisfying $\nu^{E}\left(\Gamma_{1}\right)>0, \nu^{E}\left(\Gamma_{2}\right)>0, \nu^{E}\left(\Gamma_{1} \cap \Gamma_{2}\right)=0$, the measures $\int_{\Gamma_{1}}^{\oplus} \tilde{\mu}_{E}^{\chi} d \nu^{E}(\chi)$ and $\int_{\Gamma_{2}}^{\oplus} \tilde{\mu}_{E}^{\chi} d \nu^{E}(\chi)$ are singular.

\section{Remarks:}

1) Two Cartan subalgebras $\mathcal{A}$ and $\mathcal{A}^{\prime}$ of $g$ give isomorphic Borel measure spaces $\left(\Gamma_{\mathcal{A}}, \nu^{E}\right)$ and $\left(\Gamma_{\mathcal{A}^{\prime}}, \nu^{E}\right)$; it follows that $\sqcup^{E^{\prime}}$ has the $\Gamma$-property with respect to some Cartan subalgebra if and only if $\omega^{E}$ has the $\Gamma$-property with respect to all Cartan subalgebras.

2) As $\tilde{\mu}_{E}^{\chi}$ is equivalent to the spectral measure of $\tilde{w}_{\chi}$, the $\Gamma$-property is equivalent to the fact that, for $\Gamma_{1}, \Gamma_{2}$ as above, $\int_{\Gamma_{1}}^{\oplus} \tilde{w}_{\chi} d \nu^{E}(\chi)$ and $\int_{\Gamma_{2}}^{\oplus} \tilde{w}_{\chi} d \nu \nu^{E}(\chi)$ contain no equivalent subrepresentations, i.e. are disjoints.

3) For all $\chi$ in $\Gamma_{\mathcal{A}}$ let $\alpha_{\chi}$ be the spectral measure of the character $\gamma_{\chi}$, and let $\Gamma_{1}, \Gamma_{2}$ be as in Definition 10 , let $\lambda_{i}$ be the measure $\int_{\Gamma_{i}}^{\oplus} \alpha_{\chi} d \nu^{E}(\chi), i=1,2$; the property $\Gamma$-property says that $\tilde{\mu}_{E} * \lambda_{1}$ and $\tilde{\mu}_{E} * \lambda_{2}$ are disjoints. 
Lemma 16. Let $E$ be $a(\tau, T X, F, \Lambda, d \ell)$-energy structure, let $\mathcal{D}(X, G)$ be a gauge group, and let us suppose that $\cup^{E}$ has the $\Gamma$-property. The von Neumann algebra generated by $\Pi_{A}^{E}$ contains all operators of the form $1 \otimes \Theta^{E}(u)$ and $W(u) \otimes 1, u$ in $\mathcal{D}(X, \mathcal{A})$.

Proof: Let us recall that $W(u)=\tilde{w}(\tilde{u}), \tilde{u} \in \tilde{\mathcal{D}}(X, \mathcal{A})$, and let us consider the family $\left(\tilde{w}_{\chi}\right)_{\chi \in \Gamma \wedge}$ of unitary representations of $\mathcal{D}(X, \mathcal{A})$ given in Lemma 15. One has $\Pi_{\mathcal{A}}^{E}=\int_{\Gamma}^{\oplus} \tilde{w}_{\chi} d \nu^{E}(\chi)$, and then $\Pi_{\mathcal{A}}^{E}$ is equivalent to a representation of $\mathcal{D}(X, \mathcal{A})$ into a direct integral of Hilbert spaces $\int_{\Gamma}^{\oplus} \mathcal{H}^{x} d \nu^{E}(\chi)$. Let $S$ be an operator commuting with the representation $\Pi_{\mathcal{A}}^{E}$, and let $\Gamma^{\prime}$ be a measurable subset of $\Gamma$ with $\nu^{E}\left(\Gamma^{\prime}\right)>0, \nu^{E}\left(\Gamma-\Gamma^{\prime}\right)>0$; from the $\Gamma$-property it follows that $\int_{\Gamma^{\prime}}^{\oplus} \mathcal{H}^{x} d \nu \nu^{E}(\chi)$ and its orthogonal complement $\int_{\Gamma-\Gamma^{\prime}}^{\oplus} \mathcal{H}^{x} d \nu^{E}(\chi)$ are such that the restrictions of $\pi_{A}^{E}$ to these two spaces contain no equivalent subrepresentations, and $\int_{\Gamma^{\prime}}^{\oplus} \mathcal{H}^{x} d \nu^{E}(\chi)$ is invariant by $S$. It follows that $S$ is decomposable into a direct integral with respect to $\nu^{E}$.

Let $N^{E}$ be the von Neumann algebra generated by $\Pi_{A}^{E}$. The operators $\left(1 \otimes \Theta^{E}\right)(u)$ and $(W \otimes 1)(u)$ act on each Hilbert space $\mathcal{H}^{X}$ by multiplication by bounded $\nu^{E}$-measurable functions; it follows that they commute with all decomposable operators, in particular with operators $S$ which commute with $\Pi_{\mathcal{A}}^{E}$; these operators are then in the bicommutant of $\Pi^{E} \mathcal{A}$, and then in $N^{E}$, by the von Neumann's theorem.

As a corollary one gets:

Corollary. $N^{E}$ contains all operators of multiplication by $e^{i<\cdot d u\rangle} E, u \in$ $\mathcal{D}(X, \mathcal{A})$.

We come now to the main result about irreducibility.

Theorem 3. Let $\mathcal{D}(X, G)$ be a gauge group, and let $E$ be $a(\tau, T X, F, \Lambda, d \ell)-$ energy structure. If $\mathrm{U}^{E}$ has the $\Gamma$-property, $\sqcup^{E}$ is irreducible.

Proof: We shall use the realization of $U^{E}$ in the space $L^{2}\left(\mathcal{D}_{1}^{\prime}(X, g) ; \mu_{E}\right)$ given in (16), Section 7(c).

a) Let us prove, at first, that the vacuum vector $1: \chi \rightarrow 1(\chi)=1 \forall \chi \in$ $\mathcal{D}_{1}^{\prime}(X, g)$, is cyclic for $\cup^{E}$. Let $L^{E}$ be the von Neumann algebra generated by $\sqcup^{E}$; as $\Pi_{\mathcal{A}}^{E}(u)=\sqcup^{E}(\exp u), u \in \mathcal{D}(X, \mathcal{A})$, the bicommutant of $\Pi_{\mathcal{A}}^{E}$ is contained in the bicommutant of $\sqcup^{E}$; from the corollary of Lemma 16, it follows that if $\sqcup^{E}$ has the $\Gamma$-property, $L^{E}$ contains the operators of multiplication by $\exp (i<, d u>), u \in \mathcal{D}(X, \mathcal{A})$, for any Cartan subalgebra $\mathcal{A}$ of $g$. As $g$ is the union of its Cartan subalgebras, it follows that $L^{E}$ contains all the operators of multiplication by $\exp (i<\cdot, d u>$ ) for all $u$ in $\mathcal{D}(X, g)$, and then, all the operators of the form:

$$
n_{u}^{E}(\bar{g})=\sqcup^{E}(\bar{g}) \quad e^{i\langle\cdot d u\rangle} \cdot \sqcup^{E}\left(\bar{g}^{-1}\right)
$$


with $g$ in $\mathcal{D}(X, G)$ and $u$ in $\mathcal{D}(X, g)$, i.e. all the operators of multiplication by:

$$
e^{\left.i<\cdot, V^{E}(\bar{g}) d u\right\rangle} g \in \mathcal{D}(X, G), u \in \mathcal{D}(X, g) .
$$

It follows that $L^{E}$ contains all the operators of multiplication by functions of the form:

$$
e^{i<\cdot \sum_{k=1}^{k=p} \sum_{j} E_{\left(\bar{g}_{k}\right) d u_{k}>}}
$$

with $g_{1}, \ldots, g_{p}$ in $\mathcal{D}(X, G)$ and $u_{1}, \ldots, u_{P}$ in $\mathcal{D}(X, g)$.

By the lemma 3.5 of $[\mathbf{1 7}]$ one knows that the set $\left\{V^{E}(\bar{g}) d u / \bar{g} \in \mathcal{D}(X, G), u \in\right.$ $\mathcal{D}(X, g)\}$ is total in $\mathcal{D}_{1}(X, g)$; it follows that the functions:

$$
\chi \longrightarrow n_{u}^{E}(\bar{g})(\chi),(\bar{g} u) \in \mathcal{D}(X, G) \times \mathcal{D}(X, g)
$$

constitute a total set in $L^{2}\left(\mathcal{D}_{1}^{\prime}(X, g) ; \mu_{E}\right)$.

As $U^{E}(\bar{g}) 1=e^{i<\cdot, b(\bar{g})>}$, it follows that the smallest closed subspace of $L^{2}\left(\mathcal{D}_{1}^{\prime}(X, g) ; \mu_{E}\right)$ containing the functions $\sqcup^{E}(\bar{g}) 1, g \in \mathcal{D}(X, G)$, contains the space spanned by the functions $n_{u}^{E}(\bar{g})$, and then, this space is exactly $L^{2}\left(\mathcal{D}_{1}^{\prime}(X, g)\right.$; $\mu_{E}$ ); the cyclicity of 1 is then proved.

b) It remains now to prove the irreducibility of the cyclic component of $\sqcup^{E}, \sqcup^{E}$ having the $\Gamma$-property.

Let $Q$ be an operator belonging to the commutant of $U^{E}(\mathcal{D}(X, G))$; $Q$ commutes with $\Pi_{\mathcal{A}}^{E}$ for any Cartan subalgebra $\mathcal{A}$ of $g$, and then, as it has been seen in the proof of Lemma $16, Q$ is decomposable with respect to the integral decomposition of $\Pi \Gamma_{\mathcal{A}}^{E}$ given in Lemma 15 , for any Cartan subalgebra $\mathcal{A}$ of $g$. The projection of $L^{2}\left(\mathcal{D}_{1}^{\prime}(X, g) ; \mu_{E}\right)$ onto $L^{2}\left(\mathcal{D}_{1}^{\prime}(X, \mathcal{A}) ; \mu_{E, \mathcal{A}}\right)$ being diagonalizable, $L^{2}\left(\mathcal{D}_{1}^{\prime}(X, \mathcal{A}) ; \mu_{E, A}\right)$ is invariant by $Q ;$ it follows that $Q 1$ belongs to all the spaces $L^{2}\left(\mathcal{D}_{1}^{\prime}(X, \mathcal{A}) ; \mu_{E, \mathcal{A}}\right)$ for all Cartan subalgebras $\mathcal{A}$. Owing to the semisimplicity of $g$, the intersection of all the spaces $L^{2}\left(\mathcal{D}_{1}^{\prime}(X, \mathcal{A}) ; \mu E, \mathcal{A}\right), \mathcal{A}$ running in the set of Cartan subalgebras of $g$, equals $\mathrm{Cl}$; it follows that $Q$ is a scalar operator; as 1 is a cyclic vector for $\cup^{E}$, it follows that $\sqcup^{E}$ is irreducible.

Note: The Theorem 3 is, up to now, the only way known in order to prove the possible irreducibility of the energy representations, and is substantially the method given in $[\mathbf{1 7}],[\mathbf{2}]$ in order to prove the irreducibility of $\sqcup^{E}$ with $E=\left(X, r d v^{\tau}, T X, q_{\tau}\right)$ when $\operatorname{dim}(X) \geq 3$; in the case $E=\left(X, r d v^{\tau}, F(\xi), q(\xi)\right)$ the irreducibility of $\sqcup^{E}$ when $\operatorname{dim}(X) \geq 3$ was also proved in $[9]$ in the same way. We do not know whether the $\Gamma$-property of $L^{E}$ is cquivalent to its irreducibility.

Acknowledgements. This work has to be considered as a part of a common work with Professors S. Albeverio, R. Høegh-Krohn and D. Testard who have given to me a great stimulus through many discussions. It is a pleasure for me to thank Professor L. Streit for his kind invitation and his warm hospitality at the Zentrum für interdisziplinäre Forschung, Universität Bielefeld, where this work was greatly facilitated. 


\section{References}

1. S. Albeverio, R. HøEGH-KROHN, The energy representation of Sobolev Lie groups, Comp. Math. 36 (1978), 37-52.

2. S. Albeverio, R. HøEGH-KROHN, D. TESTARD, Irreducibility and reducibility of the encrgy representation of the group of mappings of a Riemannian manifold into a compact semisimple Lie group, J. Funct. Anal. 41, 3 (1981), 378-396.

3. S. Albeverio, R. Høegh-Krohn, D. Testard, Factoriality of representations of the group of paths on $S U(n)$, Preprint $Z i F \mathbf{9}$, Bielefeld University (1984).

4. S. Albeverio, R. Høegh-Krohn, D. Testard, A.M. Vershik, Factorial representations of path groups, J. Funct. Anal. 51 (1983), 115-131.

5. A. ConNes, A survey of foliations and operator algebra in operators, algebras and applications, Proc. Symp. in Math. A.M.S 38, 1 (1982), 521-628.

6. A. GUICHARDET, "Symmetric Hibert spaces and related topics," Lect. Notes in Math. 261, Springer-Verlag, 1972.

7. R. Ismagilov, On unitary representations of the group $C_{0}(X, G), G=$ $S U(2)$, Mat. Sb. (N.S.) 100, 2 (1976), 117-131 [Russian].

8. R. ISMAGILOV, Representations of the group of smooth mappings of a segment into a compact Lie group, Funk. Anel. Ego. Pril. 15, 2 (1981), 73-74 [Russian].

9. J. MARION, Generalizad energy representations for current groups, $J$. Funct. Anal. 54, 1 (1983), 1-17.

10. J.Marion, $G$-distributions et $G$-integrales multiplicatives sur une variété, Anal. Pol. Math. 43 (1983), 71-84.

11. J. MARION, A survey on the unitary representations of gauge groups, and some remaining open questions, Conf, at the ZiF, Bielefeld University (1983), Trends and Developments in the Eighties (to appear). World Scientific Publishing Co., Singapore.

12. J. Milnor, J. Stasheff, Characteristic classes, Ann. of Math. Studies 76. Princ. Univ. Press (1974).

13. B. ReinnaRT, "Differential geometry of foliations," Springer-Verlag, Berlin-Heidelberg-New York-Tokyo, 1983.

14. N. Steenrod, "Topology of fiber bundles," Princ. Univer. Press, N.J, 1951.

15. F. TREves, "Topological vector spaces, distributions and kernels," Academic Press, New York, 1976. 
16. A.M. Vershik, I.M. GELFAND, M.I. GraEV, Representations of the group $C_{0}^{\infty}(X, G), G$ compact, Dokl. Akad. Nauk. 232 (1977), 745-748 [Russian].

17. A.M. VershiK, I.M. GElfand, M.I. Graev, Representations of the group of smooth mappings of a manifold $X$ into a compact Lie group, Comp. Math. 35 (1977), 299-336.

18. A.M. VershiK, I.M. Gelfand, M.I. Graev, Representations of the group of functions taking values in a compact Lie group, Comp. Math. 42 (1981), 217-243.

Département de Mathématique

Facuité des Sciences de Marseille-Luminy, and

Laboratoire associé au C.N.R.S. No. 225

FRANCE

Rebut el 19 de Maig de 1988 